\title{
DEEP LEARNING-BASED METHODOLOGICAL APPROACH FOR VINEYARD EARLY DISEASE DETECTION USING HYPERSPECTRAL DATA
}

\author{
Jonáš Hruška *, Telmo Adão *', Luís Pádua *, Pedro Marques *, Emanuel Peres ${ }^{*}{ }^{*}$, António Sousa * ${ }^{*}$, \\ Raul Morais *', Joaquim João Sousa *, ${ }^{\dagger}$ \\ * University of Trás-os-Montes e Alto Douro, \\ + INESC Technology and Science (INESC-TEC)
}

\begin{abstract}
Machine Learning (ML) progressed significantly in the last decade, evolving the computer-based learning/prediction paradigm to a much more effective class of models known as Deep learning (DL). Since then, hyperspectral data processing relying on DL approaches is getting more popular, competing with the traditional classification techniques. In this paper, a valid ML/DL-based works applied to hyperspectral data processing is reviewed in order to get an insight regarding the approaches available for the effective meaning extraction from this type of data. Next, a general DL-based methodology focusing on hyperspectral data processing to provide farmers and winemakers effective tools for earlier threat detection is proposed.
\end{abstract}

Index Terms - Hyperspectral data, remote sensing, agriculture, forestry, machine learning, deep learning

\section{INTRODUCTION}

Hyperspectral-based remote sensing (RS) methods consist of the image cubes acquisition where, for each of the image's spatially distributed elements, a spectrum of the energy reaching the respective sensor is measured. Hyperspectral imagery consists of hundreds or thousands of bands arranged in a narrow bandwidth $(5-20 \mathrm{~nm}$, each). After operations prior to flight and post-acquisition data pre-processing - i.e. hardware calibration, radiometric correction, ground control points in the field, etc. - collected hyperspectral data needs to be properly computed to extract relevant information through reflectance behavior discrimination. For many years, such goal has been achieved through traditional techniques that include statistical modelling over data (e.g. target detection algorithms), feature extraction for multiclass discrimination, vegetation indexes with acceptable results. Although, emerging approaches to deal with hyperspectral data processing complexity based on DL are competing with the traditional techniques in terms of accuracy [1]. Several promising results have been reported for plant identification, crop disease detection or reduction of pesticide usage (e.g. [2]-[6]).

Alongside the pointed developments in hyperspectral data processing, remote sensing progresses also brought to light Unmanned Aerial System (UAS) that are getting increasingly popular in agricultural and forestry. Plus, they represent a cost-effective and readily available tool for surveying land and crops, with the purpose of acquiring data for further analysis and to support decision-making and management processes. Meanwhile, hyperspectral sensors commonly used on satellites or manned aircraft a few years ago - are getting redesigned to be lighter and smaller and, thus, more suitable to be carried by UAVs. Considering the aforementioned factors, the main goal of this paper is a methodology proposal - refined from [7] - to support farmers and winemakers with effective tools for early threat detection in vineyards through DL-based decision support capabilities. Underlying steps include UAS-based surveying, pre-processing tasks, hyperspectral data computation and results conversion to human-readable guidelines.

\section{HYPERSPECTRAL DATA PROCESSING USING DL APPROACHES}

The natural light is primary distant source of energy in a passive RS system that reaches the Earth as a solar radiation. The reflectance spectra of vegetation canopies can be obtain by using radiative transfer models in direct mode for sensitivity analysis and or by inverted models for parameter retrieval [8]. In terms of processing the hyperspectral data, the most crucial part is classification. The traditional techniques for hyperspectral data classification are based on classifying each pixel by the spectral signatures from different bands, and the classification algorithms usually consist of parallelepiped classification, k-nearest neighbors, maximum likelihood, minimum distance and logistic regression [9]. Progresses were made through ML/DL-based pixel-wise classification. A known drawback related to such classification is that they process each pixel independently on their spatial information [10]. However, the spatial information of hyperspectral objects is as important as spectral information [11]. In fact, spatial information can make the classification more precise by reducing the uncertainty of samples [12]. Thus, the classification accuracy can be improved if the spatial information is well adopted. Hyperspectral image classification methods that use this exploitation include particularly image segmentation based methods [13], [14] and sparse representation [15][17]. To deal with the amount of bands and redundancy involved in hyperspectral data, it is very common to apply dimensionality reduction [18]. To that end, two methods are known: transformation [19] and band selection [20]. 
DL includes a class of model that is learning hierarchically deep features of input data with very deep NN [21]. In [22], [23] is noted that deep models provide more accurate approximation to nonlinear functions than shallow models. Deep neural network architecture typically comprise Deep Belief Networks (DBNs), Deep Boltzmann Machines (DBMs), Stacked Autoencoder (SAE) and stacked denoising autoencoders [21].

For example, in [24], a framework that joins dimension reduction and DL to allow hyperspectral image classification based on spectral-spatial features is proposed. Discriminant algorithms for spectral features and Convolutional Neural Networks $(\mathrm{CNN})$ to deal with spatial features are at the basis of this proposal that is pointed out as an outperforming framework comparatively to commonly used methods for hyperspectral image classification. For saliency detection regarding to band selection in hyperspectral imaging (HSI), a manifold ranking approach - that is also extended to DLis presented in [25]. The spectral-spatial residual network found in [26] consists of a supervised DL framework that is capable of discriminating features from abundant spectral signatures and spatial contexts in a HSI (provided as input and without the need for feature engineering). In [27], both attribute profile and DL approaches were merged with the goal of classifying HSI. Profiling working together with CNN showed better results than using each of the involved approaches individually. Also, the need for lighter network architectures and deep network types was highlighted. Even for anomaly detection, CNN demonstrated to outperform classical methods such as Reed-Xiaoli (RX)-based approaches [28]

In [29] a novel Recurrent Neural Networks (RNN) model was presented, inspired from the observation that hyperspectral pixels can be regarded as sequential data. The proposed model achieved higher accuracy than Support Vector Machine (SVM) with Radial Basis Function (RBF) and CNN for hyperspectral image classification. In [30] were explored CNNs for HSI classification with a great results. Hyperspectral data classification method using deep features extracted by SAEs was proposed by [21]. The presented SAE with Logistic Regression (SAE-LR) showed statistically higher accuracy than RBF-SVM, considered as conventional classifier. In [17] an active set algorithm for learning relevant features in spatial-spectral HIS classification was presented. The effectiveness and efficiency were proven by testing the approach in analysis of four hyperspectral classification scenarios. Supervised 3-D DL framework for spectral-spatial representation learning and HSI classification was presented in [26].

As a concrete application example, Castro et al. [31] investigated the detection of cruciferous weeds in wheat and broad bean crop using hyperspectral and multispectral readings. To detect the cruciferous weed reflectance differences, three classifications methods were applied: stepwise discriminant (STEPDISC) analysis and two neural networks, specifically, Multilayer Perceptron (MLP) and RBF.

With a broader insight in hyperspectral processing though ML/DL, next section focuses on the proposal of a DL-based methodology for early threat detection in vine cultures.

\section{DL-BASED ARCHITECTURAL PROPOSAL FOR VINEYARD DISEASE DETECTION}

Considering the key-knowledge that will extract relevant information from the acquired hyperspectral data, the remainder of this paper is dedicated to the proposal of a methodology - depicted in Figure 1 - that explores UASbased high-resolution spectroscopy for vineyard diseases early detection and further monitoring.

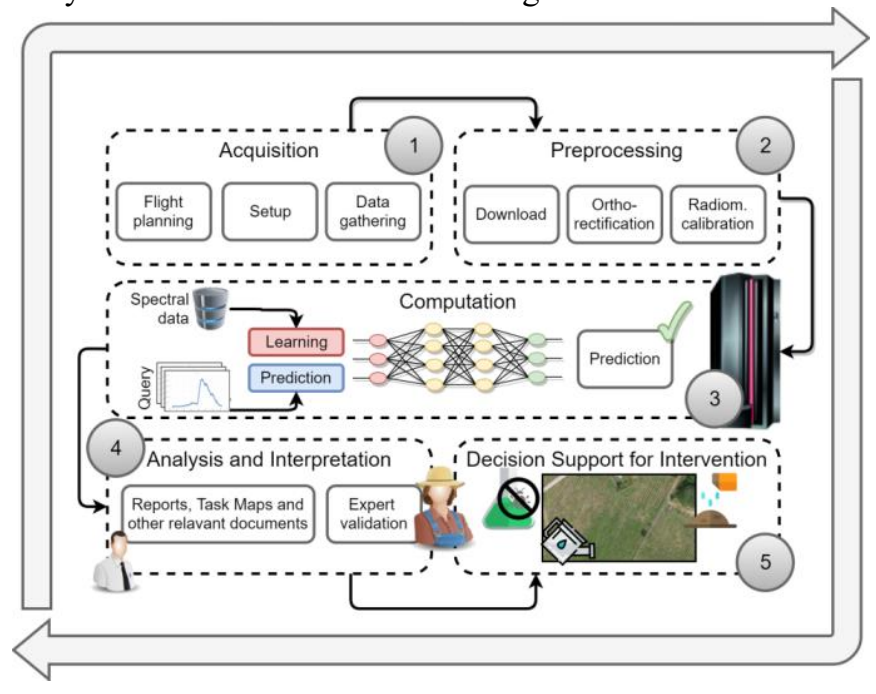

\section{Figure 1 Methodology proposal for a full-stack vineyard early} disease detection and monitoring system.

The underlying processes rely on the following: (1) data acquisition, (2) pre-processing, (3) data computation (4) analysis and interpretation and (5) decision support for intervention. Data acquisition refers to the auto-pilot mode flight campaigns carried out with UAS to gather hyperspectral data with a lightweight pushbroom VNIR hyperspectral sensor with on-board data processing, data storage of 480GB, GPS and IMU for small UAV applications. Spectral range of the sensor is (400-1000 nm) with 270 bands. Next, acquired data must undergo a preprocessing step that usually consists of calibrating imagery regarding radiometry, atmospheric noise, etc., as well as performing orthorectification - required when a pushbroom sensor is used, as it is the case - for spatial correction purposes. Afterwards, data computation is responsible for highlighting the occurrence of diseases, using DL. A data set must be initially used to teach the machine - computer-based entity - about the features that should be considered to discriminate healthiness from abnormal conditions and also for distinguishing diseases. After this learning step, the machine will develop processes by itself to predict the phytosanitary status in new querying data. Intelligent 
algorithms have already been applied to hyperspectral data [32] attesting the feasibility of this combination. Just like for statistical-based hyperspectral data manipulation, some engines and libraries (e.g. Tensorflow) to support the development of ML applications - as the one that is being proposed - are available. Outcome is then analyzed and interpreted by an expert who can adjust some parameters (for example, perform corrections on undetected disease areas and deleting parts wrongly marked with infection) to correct farmer's guiding files (reports, task maps, etc.). Eventually, these rectifications might be used to fine-tune the computational approach parameters for performance improvement purposes. Lastly, validated data is delivered to the farmer who will be able to perform field interventions with decision support provided by, for example: (1) heat maps properly subtitled to facilitate the identification of problems in a terrain parcel, from an aerial point of view; and (2) a set of instructions detailing the most proper treatments.

Sustainable agricultural practices and yield improvement through the proper administration of phytosanitary products are the main goals of this methodology. However, other goals will be pursued, such as support decision for proper water irrigation (according to hydric stress) and fertilizer application (considering nitrogen concentrations). Finally, multi-temporal data sets shall be considered for predicting yields and enhance agricultural practices upon the different vineyard development stages.

\section{CONCLUSION}

DL for hyperspectral data classification seems to be effective method because of its already proven ability to deal with high dimensionality and to discriminate features. Even though very robust labeled dataset for training is needed for accurate results, the potential in combining hyperspectral data with DL is enormous and, thus, desirable to implement the proposed methodology. Applying of DL approach to hyperspectral data processing has already started in our team and the results will be presented in upcoming works.

\section{ACKNOWLEDGMENTS}

This work was financed by the European Regional Development Fund (ERDF) through the Operational Programme for Competitiveness and Internationalisation COMPETE 2020 under the PORTUGAL 2020 Partnership Agreement, and through the Portuguese National Innovation Agency (ANI) as a part of project "PARRA - Plataforma integrAda de monitoRização e avaliação da doença da flavescência douRada na vinhA" ( $\mathrm{N}^{\circ}$ 3447) and ERDF and North 2020 - North Regional Operational Program, as part of project "INNOVINEandWINE - Vineyard and Wine Innovation Platform" (NORTE-01-0145-FEDER-000038).

\section{REFERENCES}

[1] T. Adão et al., "Hyperspectral Imaging: A Review on UAV-Based Sensors, Data Processing and Applications for Agriculture and Forestry," Remote Sens., vol. 9, no. 11, p. 1110, Oct. 2017.

[2] J. Amara, B. Bouaziz, and A. Algergawy, “A Deep Learning-based Approach for Banana Leaf Diseases Classification.," in BTW (Workshops), 2017, pp. 7988.

[3] A. Fuentes, S. Yoon, S. C. Kim, and D. S. Park, “A Robust Deep-Learning-Based Detector for Real-Time Tomato Plant Diseases and Pests Recognition," Sensors, vol. 17, no. 9, Sep. 2017.

[4] A. Lowe, N. Harrison, and A. P. French, "Hyperspectral image analysis techniques for the detection and classification of the early onset of plant disease and stress," Plant Methods, vol. 13, Oct. 2017.

[5] S. Sladojevic, M. Arsenovic, A. Anderla, D. Culibrk, and D. Stefanovic, "Deep Neural Networks Based Recognition of Plant Diseases by Leaf Image Classification," Computational Intelligence and Neuroscience, 2016. [Online]. Available: https://www.hindawi.com/journals/cin/2016/3289801/ . [Accessed: 09-Jan-2018].

[6] G. Wang, Y. Sun, and J. Wang, "Automatic ImageBased Plant Disease Severity Estimation Using Deep Learning," Computational Intelligence and Neuroscience, 2017. [Online]. Available: https://www.hindawi.com/journals/cin/2017/2917536/ . [Accessed: 09-Jan-2018].

[7] T. Adão, E. Peres, L. Pádua, J. Hruška, J. Sousa, and R. Morais, "UAS-based hyperspectral sensing methodology for continuous monitoring and early detection of vineyard anomalies," 28-Jun-2017.

[8] H. Bach and W. Verhoef, "Sensitivity studies on the effect of surface soil moisture on canopy reflectance using the radiative transfer model GeoSAIL," in IGARSS 2003. 2003 IEEE International Geoscience and Remote Sensing Symposium. Proceedings (IEEE Cat. No.03CH37477), 2003, vol. 3, pp. 1679-1681.

[9] G. M. Foody and A. Mathur, "A relative evaluation of multiclass image classification by support vector machines," IEEE Trans. Geosci. Remote Sens., vol. 42, no. 6, pp. 1335-1343, Jun. 2004.

[10] X. Ma, J. Geng, and H. Wang, "Hyperspectral image classification via contextual deep learning," EURASIP J. Image Video Process., vol. 2015, no. 1, p. 20, Dec. 2015.

[11] R. M. Willett, M. F. Duarte, M. A. Davenport, and R. G. Baraniuk, "Sparsity and Structure in Hyperspectral Imaging : Sensing, Reconstruction, and Target Detection," IEEE Signal Process. Mag., vol. 31, no. 1, pp. 116-126, Jan. 2014.

[12] P. Ghamisi, J. A. Benediktsson, and J. R. Sveinsson, "Automatic Spectral \#x2013; Spatial Classification 
Framework Based on Attribute Profiles and Supervised Feature Extraction," IEEE Trans. Geosci. Remote Sens., vol. 52, no. 9, pp. 5771-5782, Sep. 2014.

[13] Y. Tarabalka, J. A. Benediktsson, J. Chanussot, and J. C. Tilton, "Multiple Spectral \#x2013;Spatial Classification Approach for Hyperspectral Data," IEEE Trans. Geosci. Remote Sens., vol. 48, no. 11, pp. 4122-4132, Nov. 2010.

[14] Z. Miao and W. Shi, "A New Methodology for Spectral-Spatial Classification of Hyperspectral Images," Journal of Sensors, 2016. [Online]. Available: https:/www.hindawi.com/journals/js/2016/1538973/. [Accessed: 05-Nov-2017].

[15] H. Zhang, J. Li, Y. Huang, and L. Zhang, “A Nonlocal Weighted Joint Sparse Representation Classification Method for Hyperspectral Imagery," IEEE J. Sel. Top. Appl. Earth Obs. Remote Sens., vol. 7, no. 6, pp. 2056-2065, Jun. 2014.

[16] Y. Chen, N. M. Nasrabadi, and T. D. Tran, "Hyperspectral Image Classification Using Dictionary-Based Sparse Representation," IEEE Trans. Geosci. Remote Sens., vol. 49, no. 10, pp. 3973-3985, Oct. 2011.

[17] D. Tuia, R. Flamary, and N. Courty, "Multiclass feature learning for hyperspectral image classification: sparse and hierarchical solutions," ISPRS J.

Photogramm. Remote Sens., vol. 105, pp. 272-285, Jul. 2015.

[18] L. Lin and X. Song, "Using CNN to Classify Hyperspectral Data Based on Spatial-spectral Information," in Advances in Intelligent Information Hiding and Multimedia Signal Processing, Springer, Cham, 2017, pp. 61-68.

[19] L. M. Bruce, C. H. Koger, and J. Li, "Dimensionality reduction of hyperspectral data using discrete wavelet transform feature extraction," IEEE Trans. Geosci. Remote Sens., vol. 40, no. 10, pp. 2331-2338, Oct. 2002.

[20] C.-I. Chang, Q. Du, T.-L. Sun, and M. L. G. Althouse, "A joint band prioritization and band-decorrelation approach to band selection for hyperspectral image classification," IEEE Trans. Geosci. Remote Sens., vol. 37, no. 6, pp. 2631-2641, Nov. 1999.

[21] Y. Chen, Z. Lin, X. Zhao, G. Wang, and Y. Gu, "Deep Learning-Based Classification of Hyperspectral Data," IEEE J. Sel. Top. Appl. Earth Obs. Remote Sens., vol. 7, no. 6, pp. 2094-2107, Jun. 2014.

[22] I. Sutskever and G. E. Hinton, "Deep, narrow sigmoid belief networks are universal approximators," Neural Comput., vol. 20, no. 11, pp. 2629-2636, Nov. 2008.

[23] N. L. Roux and Y. Bengio, "Deep Belief Networks Are Compact Universal Approximators," Neural Comput., vol. 22, no. 8, pp. 2192-2207, Aug. 2010.
[24] W. Zhao and S. Du, "Spectral \#x2013;Spatial Feature Extraction for Hyperspectral Image Classification: A Dimension Reduction and Deep Learning Approach," IEEE Trans. Geosci. Remote Sens., vol. 54, no. 8, pp. 4544-4554, Aug. 2016.

[25] Q. Wang, J. Lin, and Y. Yuan, "Salient Band Selection for Hyperspectral Image Classification via Manifold Ranking," IEEE Trans. Neural Netw. Learn. Syst., vol. 27, no. 6, pp. 1279-1289, Jun. 2016.

[26] Z. Zhong, J. Li, Z. Luo, and M. Chapman, "SpectralSpatial Residual Network for Hyperspectral Image Classification: A 3-D Deep Learning Framework," IEEE Trans. Geosci. Remote Sens., vol. PP, no. 99, pp. 1-12, 2017.

[27] E. Aptoula, M. C. Ozdemir, and B. Yanikoglu, "Deep Learning With Attribute Profiles for Hyperspectral Image Classification," IEEE Geosci. Remote Sens. Lett., vol. 13, no. 12, pp. 1970-1974, Dec. 2016.

[28] W. Li, G. Wu, and Q. Du, "Transferred Deep Learning for Anomaly Detection in Hyperspectral Imagery," IEEE Geosci. Remote Sens. Lett., vol. 14, no. 5, pp. 597-601, May 2017.

[29] L. Mou, P. Ghamisi, and X. X. Zhu, "Deep Recurrent Neural Networks for Hyperspectral Image Classification," IEEE Trans. Geosci. Remote Sens., vol. 55, no. 7, pp. 3639-3655, Jul. 2017.

[30] W. Hu, Y. Huang, L. Wei, F. Zhang, and H. Li, “Deep Convolutional Neural Networks for Hyperspectral Image Classification," Journal of Sensors, 2015. [Online]. Available: https://www.hindawi.com/journals/js/2015/258619/. [Accessed: 02-Nov-2017].

[31] A.-I. de Castro, M. Jurado-Expósito, M.-T. GómezCasero, and F. López-Granados, "Applying Neural Networks to Hyperspectral and Multispectral Field Data for Discrimination of Cruciferous Weeds in Winter Crops," Sci. World J., vol. 2012, May 2012.

[32] A. Plaza et al., "Recent advances in techniques for hyperspectral image processing," Remote Sens. Environ., vol. 113, pp. S110-S122, Sep. 2009. 